\title{
Cavitary Plaques in Otospongiosis: CT Findings and Clinical Implications
}

\author{
(D). Puac, (D) A. Rodríguez, (D) H.-C. Lin, (D) V. Onofrj, (D)F.-C. Lin, (D).-C. Hung, (D) C. Zamora, and (D) M. Castillo
}

\begin{abstract}
BACKGROUND AND PURPOSE: Cavitary plaques have been reported as a manifestation of otospongiosis. They have been related to third window manifestations, complications during cochlear implantation, and sensorineural hearing loss. However, their etiology and clinical implications are not entirely understood. Our purpose was to determine the prevalence, imaging findings, and clinical implications of cavitary plaques in otospongiosis.
\end{abstract}

MATERIALS AND METHODS: We identified patients with otospongiosis at a tertiary care academic medical center from January 2012 to April 2017. Cross-sectional CT images and clinical records of 47 patients ( 89 temporal bones) were evaluated for the presence, location, and imaging features of cavitary and noncavitary otospongiotic plaques, as well as clinical symptoms and complications in those who underwent cochlear implantation.

RESULTS: Noncavitary otospongiotic plaques were present in 86 (97\%) temporal bones and cavitary plaques in 30 (35\%). Cavitary plaques predominated with increasing age (mean age, 59 years; $P=.058$ ), mostly involving the anteroinferior wall of the internal auditory canal $(P=$ $.003)$, and their presence was not associated with a higher grade of otospongiosis by imaging $(P=.664)$ or with a specific type of hearing loss $(P=.365)$. No patients with cavitary plaques had third window manifestations, and those with a history of cochlear implantation $(n=$ 6) did not have complications during the procedure.

CONCLUSIONS: Cavitary plaques occurred in one-third of patients with otospongiosis. Typically, they occurred in the anteroinferior wall of the internal auditory canal. There was no correlation with the degree of otospongiosis, type of hearing loss, or surgical complications. Cavitary plaques tended to present in older patients.

ABBREVIATION: IAC = internal auditory canal

O tospongiosis is an osteodystrophic disorder of the otic capsule that results in acquired hearing loss with a peak onset in the third decade. ${ }^{1-3}$ It is believed to originate in cartilaginous remnants within the endochondral layer of the otic capsule, which are

Received September 25, 2017; accepted after revision February 3, 2018.

From the Division of Neuroradiology (P.P., A.R., V.O., S.-C.H., C.Z., M.C.), Department of Radiology, University of North Carolina School of Medicine, Chapel Hill, North Carolina; Radiology Department (H.-C.L.), Cathay General Hospital, Taipei, Taiwan; and Department of Biostatistics and North Carolina Translational and Clinical Sciences Institute (F.-C.L.), University of North Carolina at Chapel Hill, Chapel Hill, North Carolina.

We acknowledge the editorial assistance of the NC Translational and Clinical Sciences Institute, which is supported by the National Center for Advancing Translational Sciences, National Institutes of Health, through grant award No. ULITR00111. Please address correspondence to Paulo Puac, MD, MSc, Neuroradiology Research Fellow, Department of Radiology, University of North Carolina School of Medicine, Chapel Hill, CB 7510, Room 2034, Old Infirmary Building, 101 Manning Dr, Chapel Hill, NC 27599-7510; e-mail: puacpaulo@gmail.com

- Indicates open access to non-subscribers at www.ajnr.org

7 Indicates article with supplemental on-line photo.

http://dx.doi.org/10.3174/ajnr.A5613 replaced by foci of more vascular bone (otospongiosis) that ultimately becomes highly calcified and sclerotic (otosclerosis). ${ }^{1,2,4-7}$

Otospongiosis manifests clinically when the lesion enlarges, encroaches on the stapedial annular ligament, and causes fixation of the stapes with resultant conductive hearing loss. If the lesion progresses to involve the cochlea, the result is irreversible sensorineural hearing loss or mixed hearing loss.

The formation of cavitary plaques in otospongiosis has been reported as a focal low-attenuation notch or diverticulum, most commonly located along the anteroinferior wall of the internal auditory canal (IAC). ${ }^{1,7-11}$ Recently, isolated IAC diverticula have been associated with a different pattern of hearing loss than that seen in classic otospongiosis. ${ }^{8}$ However, the prevalence of such diverticula or cavitary changes and their clinical implications in the setting of lesion grade or extent is not completely understood. Cavitary plaques are also thought to be a possible cause of "third window lesions," secondary to involvement of the endosteal layer of the bony labyrinth, and previous reports have also suggested 
that they may lead to CSF gushing or electrode misplacement during cochlear implantation. ${ }^{1,4,7,12}$

Therefore, the purpose of this study was the following: 1) to determine the prevalence of cavitary plaques in otospongiosis and correlate them with lesion grade, 2) describe the imaging findings and locations within the temporal bone, and 3 ) determine the clinical significance in terms of a pattern of hearing loss, third window manifestations, and complications after cochlear implantation.

\section{MATERIALS AND METHODS}

The radiology data base of the University of North Carolina was searched for all patients with a clinical diagnosis of otospongiosis who underwent a CT study from January 2012 to April 2017. The study was approved by our institutional review board, and because of its retrospective nature, informed patient consent was waived. Forty-seven patients were included.

\section{Patient Selection}

Inclusion criteria were adult patients with imaging and/or clinical findings consistent with unilateral or bilateral otospongiosis. Clinical criteria were a history of progressive hearing loss with pure tone audiometry showing conductive hearing loss with an air-bone gap of $>20 \mathrm{~dB}$ above a normal adult hearing level and with a perceptive hearing loss of $<35 \mathrm{~dB}$ above the normal adult hearing level in the range of $0.5,1,2$, and $4 \mathrm{kHz}$. Imaging findings included areas of demineralization appearing as radiolucency on CT (otospongiotic plaques) involving the otic capsule, with or without complete or partial obliteration of the oval or round windows.

Exclusion criteria included comorbid middle or inner ear pathology based on clinical history and imaging findings, including cholesteatoma, tympanic membrane perforation, ossicular dislocation, osteogenesis imperfecta, Paget disease, otosyphilis, postsurgical changes, and patients with inconclusive clinical and/or imaging findings of otospongiosis. To analyze the type of hearing loss and third window manifestations, we excluded patients who presented with a concomitant history of Menière disease, semicircular canal dehiscence, enlarged vestibular aqueduct, and perilabyrinthine fistula.

\section{Clinical Findings}

Medical charts were reviewed, and we recorded the following data for each patient: 1) age, sex, type of hearing loss, classified as conductive hearing loss, sensorineural hearing loss, and mixed hearing loss; 2) the presence of third window abnormalities defined as sound-induced vertigo, dizziness, nausea, or eye movements (Tullio phenomenon); and 3) cochlear implantation and its possible complications such as CSF gusher and electrode misplacement in cavitary formations.

\section{CT Studies}

High-resolution scans of the temporal bones were performed on 128- or 64-slice multidetector CT scanners with 0.6-mm collimation, 0.55 pitch, $320 \mathrm{mAs}$, and $120 \mathrm{kV}$ (peak); or conebeam CT with $0.6-\mathrm{mm}$ collimation, $140 \mathrm{mAs}$, and $90 \mathrm{kVp}$. Axial images parallel to the lateral semicircular canal were obtained. Coronal reformatted images were created perpendicular to the axial images. Images with extensive motion or implant artifacts were ex-

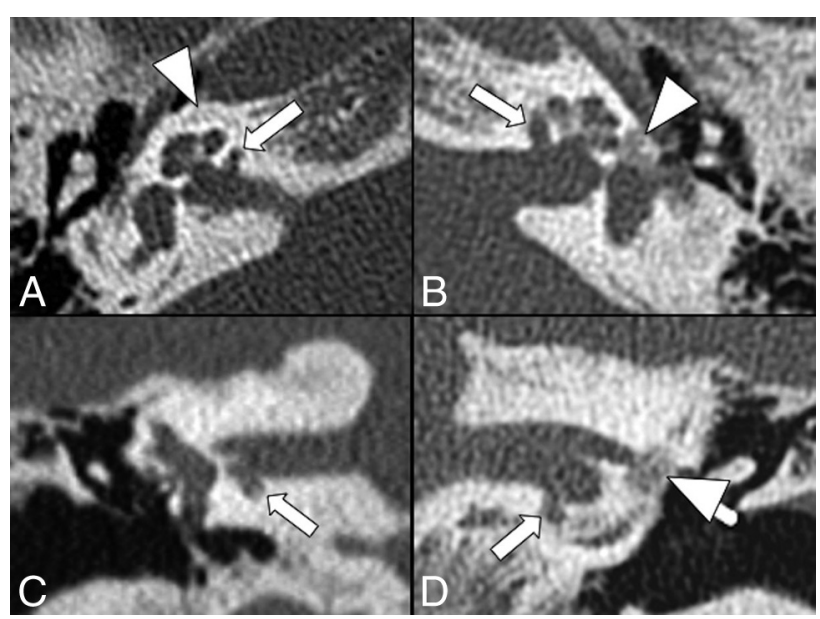

FIG 1. Bilateral cavitary plaques. Axial (top row) and coronal (bottom row) CT scans show the presence of abnormal CSF-attenuating focal lesions (arrows) involving the anterior and inferior walls of the IAC next to the basal turn of the cochlea. Additionally, there are noncavitary plaques (arrowheads) around the cochlea on the right $(A)$ and at the fissula ante fenestram on the left $(B$ and $D)$.

cluded from the study. All studies were performed without intravenous contrast administration.

\section{Image Evaluation}

Eighty-nine temporal bones from 47 patients were analyzed by 1 neuroradiology fellow (P.P.) and verified by 1 neuroradiologist with 3 years of experience reading temporal bone $\mathrm{CT}$ images and with a Certificate of Added Qualification in neuroradiology (C.Z.), both blinded to clinical findings. Findings on CT were classified into 2 groups: 1 ) otospongiotic plaques (noncavitary plaques), and 2) cavitary plaques. Otospongiotic plaques (areas of demineralization appearing as radiolucency on CT) were classified according to the Symons/Fanning classification into the following: grade 0 , no findings; grade 1, solely fenestral (fissula ante fenestram), evidence of a thickened stapes footplate, and/or decalcified, narrowed, or enlarged round or oval windows; grade 2, patchy localized cochlear disease (with or without fenestral involvement); and grade 3 , diffuse confluent cochlear involvement of the otic capsule (with or without fenestral involvement). ${ }^{11}$

Cavitary plaques were defined as focal, well-delineated, lowattenuating foci similar to CSF (Fig 1). Their location was classified as the following: zone 1 (a region anterior to the oval window), zone 2 (pericochlear region), zone 3 (anteroinferior wall of the IAC), zone 4 (posterior wall of the IAC), and zone 5 (round window) (On-line Figure).

Endosteal involvement was defined as invasion of the cavitary plaque into the endosteal layer of the labyrinth. Communication between IAC cavitary plaques and CSF was determined by lack of a normal bone between the cavity and the IAC (Fig 2).

Hounsfield units from the center of otospongiotic and cavitary plaques were measured by placing ROIs according to the size of the lesion. Studies acquired with conebeam CT were excluded for this analysis. $(n=5)$.

\section{Statistical Analysis}

Descriptive statistics were used to determine the prevalence of cavitary plaques in otospongiosis. To account for multiple out- 


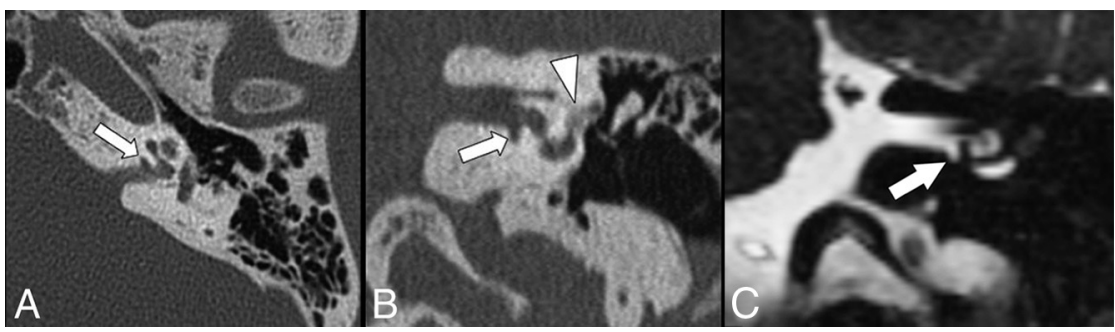

FIG 2. Axial $(A)$ and coronal (B) CT images show the presence of a cavitary plaque (arrows) involving the anterior and inferior walls of the IAC next to the basal turn of the cochlea. Additionally, there is an otospongiotic plaque (arrowhead) at the fissula ante fenestram. Coronal CISS MR image $(C)$ demonstrates a clear communication between the cavity and CSF of the IAC.

\section{Imaging Findings}

A total of 89 temporal bones were analyzed. Three had normal CT findings (otospongiosis grade 0), though history and clinical parameters were consistent with otospongiosis. Eighty-six temporal bones had classic imaging findings of otospongiosis (noncavitary plaques) and $30(35 \%)$ of them also presented cavitary plaques. Cavitary plaques were therefore never seen in isolation.
Table 1: Demographic characteristics

\begin{tabular}{lccr}
\hline \multicolumn{1}{c}{ Characteristic } & $\begin{array}{c}\text { Otospongiosis } \\
\text { and Cavitary } \\
\text { Plaques }(\boldsymbol{n}=\mathbf{3 0})\end{array}$ & $\begin{array}{c}\text { Otospongiosis } \\
\text { Only } \\
(\boldsymbol{n}=\mathbf{5 6})\end{array}$ & $\begin{array}{c}\boldsymbol{P} \\
\text { Value }\end{array}$ \\
\hline Median age (yr) & 59 & 51 & .058 \\
Temporal bone involvement & & & .273 \\
$\quad$ Unilateral (No.) (\%) & $12(40)$ & $14(25)$ & \\
$\quad$ Bilateral (No.) (\%) & $18(60)$ & $42(75)$ & \\
Hounsfield unit & 115 & 953 & $<.001$ \\
\hline
\end{tabular}

Table 2: Location of cavitary plaques in otospongiosis

\begin{tabular}{lcc}
\hline & \multicolumn{2}{c}{ Cavitary } \\
\cline { 2 - 3 } \multicolumn{1}{c}{ Location } & No. & $\%$ \\
\hline IAC & 29 & 93.54 \\
Anteroinferior wall & 28 & 90.34 \\
Posterior wall & 1 & 3.2 \\
Non-IAC & 2 & 6.46 \\
Pericochlear & 1 & 3.2 \\
Fenestral & 1 & 3.2 \\
\hline
\end{tabular}

comes from a patient, we used a generalized linear (logistic) mixed-effects model with a random intercept to determine the association of cavitary plaques with the degree of otospongiosis, type of hearing loss, third window manifestations, and complications during cochlear implantation, as well as relationships between cavitary plaques and the patient's age, sex, and Hounsfield units. The aforementioned independent variables were coded according to their data type. Type III tests for fixed effects were used to determine the overall statistical significance of the variable. $P$ values $<.05$ were considered significant. SAS 9.4 (SAS Institute, Cary, North Carolina) was used to generate descriptive statistics as well generalized linear mixed models using PROC GLIMMIX (https://support.sas.com/documentation/cdl/en/statug/63033/ HTML/default/viewer.htm\#glimmix_toc.htm).

\section{RESULTS}

\section{Patient Profile}

We identified 47 patients with otospongiosis. The mean patient age was $55 \pm 14$ years (range, $28-83$ years). Twenty-five patients (53\%) were women, and $22(47 \%)$ were men.

Of the 47 patients, 42 (89\%) had otospongiosis bilaterally, and $5(11 \%)$, unilaterally. Of the 5 patients with unilateral otospongiosis, their contralateral temporal bones were excluded because they had normal audiometry findings and no findings on $\mathrm{CT}$.

\section{Otospongiotic Plaques}

Grade 1 otospongiosis was the most common presentation in $49.4 \%(n=44)$ of the temporal bones followed by grade 3 in $29.2 \%(n=26)$. Hounsfield units from the center of the otospongiotic plaques were measured in 81 of 86 temporal bones ( 5 temporal bones with conebeam CT were excluded), resulting in Hounsfield units of $953 \pm 278$.

\section{Otospongiotic and Cavitary Plaques}

From the 30 temporal bones with cavitary plaques, 18 (60\%) showed bilateral, and 12 (40\%), unilateral cavitary changes $(P=.273)$ (Table $1)$. Regarding the number of cavitary plaques per temporal bone, 96.7\% ( $n=29)$ of temporal bones had a single cavitary plaque and only 1 (3.3\%) had 2 cavitary lesions. Of 31 cavitary plaques, $93.5 \%$ $(n=29)$ were in the IACs, and $6.5 \%(n=2)$, within the otic capsule $(P=.003)$ (Table 2). The anteroinferior wall of the IAC was the most common location for the presence of cavitary plaques (Fig 1).

The presence of cavitary plaques was not associated with a higher grade of otospongiosis by imaging ( $P=.664$ ). Otospongiosis with cavitary changes tended to present in patients older (mean age, $59 \pm 11$ years) than those without cavities (mean age, $51 \pm 15$ years), though the difference was not statistically significant $(P=.058)$ (Table 1$)$. There was no statistically significant association between the presence of cavitary plaques and sex $(P=$ $.667)$.

Of the 29 cavitary plaques located in the IACs, 97\% $(n=28)$ showed direct communication with the CSF space of the IAC (Fig 2). Endosteal involvement was seen affecting the basal turn of the cochlea in $3(10 \%)$ temporal bones with cavitary plaques.

The average length and width of the cavitary plaques were $4.44 \pm 2.32$ and $1.19 \pm 0.45 \mathrm{~mm}$, respectively. There was a statistically significant difference in the mean Hounsfield unit value between the noncavitary and cavitary plaques, $953 \pm 278$ versus $115 \pm 75$, respectively $(P<.001)$.

\section{Clinical Findings}

The type of hearing loss was analyzed in 83 of 89 temporal bones (6 temporal bones had an associated history of Menière disease). In the group of patients with cavitary changes $(n=29)$, sensorineural (41.4\%) and mixed hearing loss (41.4\%) were most common. Mixed hearing loss (51.9\%) was the most common type in the group without cavities (Table 3 ). The presence of cavitary plaques showed no significant association with a specific type of hearing loss $(P=.365)$. 
Table 3: Hearing loss according to type of otospongiosis

\begin{tabular}{lccc}
\hline & $\begin{array}{c}\text { Otospongiosis } \\
\text { and Cavitary } \\
\text { Plaques }\end{array}$ & $\begin{array}{c}\text { Otospongiosis } \\
\text { Only }\end{array}$ & $\begin{array}{c}\boldsymbol{P} \\
\text { Value }\end{array}$ \\
\hline $\begin{array}{l}\text { Type of hearing loss (No.) } \\
\text { Conductive }\end{array}$ & $5(6 \%)$ & $13(15.7 \%)$ & .365 \\
Sensorineural & $12(14.5 \%)$ & $13(15.7 \%)$ & \\
Mixed & $12(14.5 \%)$ & $28(33.7 \%)$ & \\
\hline
\end{tabular}

None of the temporal bones with otospongiosis, either with or without cavitary changes, had a clinical history of third window manifestations. There were 6 temporal bones with cavitary plaques in patients who underwent cochlear implantation, none of whom had procedural complications such as CSF gusher or misplacement of electrodes into the cavitary plaques.

\section{DISCUSSION}

The first report of cavitary plaques in otospongiosis was published by Schuknecht and Kirchner in $1974^{13}$; they described a case showing a large cavity surrounding the middle and apical turns of the cochlea. After this initial case, cavitary changes were mainly described in reports that included an average of $1-2$ cases. ${ }^{1,7,9,13}$ An abstract in 2012 reported 32 cases of cavitary changes from a series of 147 temporal bones with a history of hearing loss, in which lesions were referred to as diverticula. ${ }^{10}$ However, imaging features were not described, and to the best of our knowledge, a full article with the details of this work has not been published in the English literature.

In 2017, Pippin et $\mathrm{al}^{8}$ reported a cavitary plaque prevalence of $18 \%$ among 66 temporal bones with otospongiosis. Our prevalence was higher at $35 \%$, which could be related to a larger sample of temporal bones with otospongiosis in our study $(n=86)$ as well as possible variations in referral bias at both institutions.

In our study, we identified 31 cavitary plaques among 30 temporal bones, and none of the cavitary plaques were seen in isolation (ie, they were associated with classic findings of otospongiosis in all cases). This observation differs from that of Pippin et al, ${ }^{8}$ who reported 57 temporal bones with cavitary plaques as an isolated finding among 807 patients. This difference could be explained by our smaller sample size and our focusing on patients with clinical and/or imaging findings of otospongiosis, whereas Pippin et al analyzed patients regardless of diagnosis. Hoeberigs et $\mathrm{al}^{10}$ in 2012 reported only 2 temporal bones with isolated cavitary plaques among 222 temporal bones in patients with conductive or mixed hearing loss. However, as mentioned before, this was an abstract, and the details of the study have not been published.

Increased prevalence of cavitary plaques has been reported in patients with greater degrees of otospongiosis by CT (grade 3), suggesting that they may be a manifestation of severe disease. ${ }^{10}$ However, in our study, we found that cavitary plaques tended to be more common in grade 1 otospongiosis (16.9\%), followed by grade $3(10.1 \%)$, and were not significantly associated with the degree of otospongiosis $(P=.365)$. These results suggest that the formation of cavities represents an additional manifestation in the dynamic process of otospongiosis.

The mean age of patients with cavitary plaques ( $59 \pm 11$ years) tended to be higher than that of patients with noncavitary plaques (51 \pm 15 years) $(P=.058)$. A similar outcome was seen by Pippin et $\mathrm{al},{ }^{8}$ who found that patients with cavitary plaques were signifi-

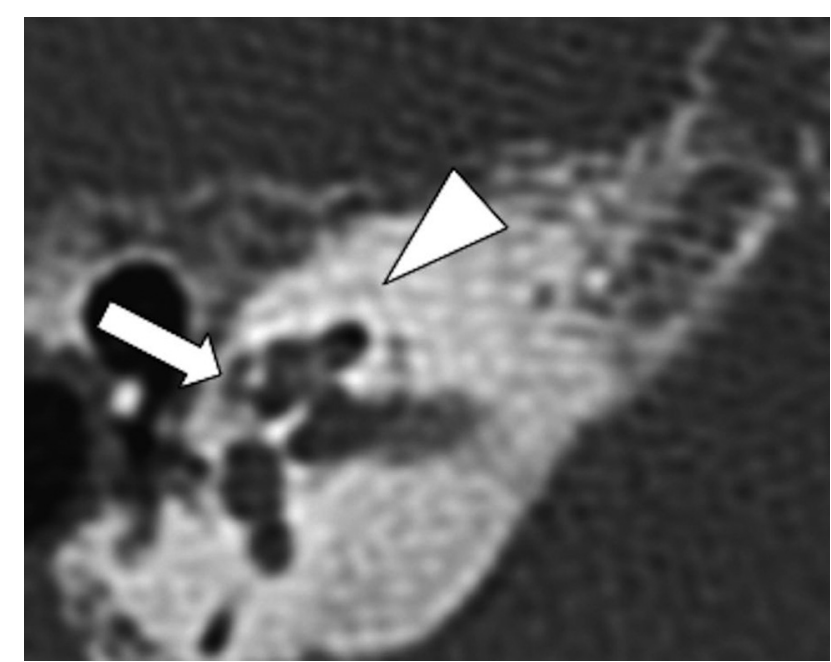

FIG 3. Axial CT scan shows the presence of a cavitary plaque involving the pericochlear region (arrow). Note the attenuation of the cavitary plaque, similar to the IAC. Additionally, there are noncavitary otospongiotic plaques surrounding the otic capsule (arrowhead).

cantly older (61 years of age) than those without cavities ( 52 years of age). Two growth patterns have been identified in otospongiotic plaques: One grows for a short time and then becomes inactive. The other pattern shows continued growth and progression throughout life. ${ }^{14}$ Because most cases of cavitary plaques were seen in patients with a long-standing diagnosis of otospongiosis, it is possible that cavitation may belong to the second growth pattern and present in older individuals, but this possibility remains uncertain.

To assess the location of cavitary plaques, we evaluated the sites within the temporal bone that have been most commonly reported in the literature. . $7,9,10,15$ Our analysis found that the walls of the IAC were the sites most commonly affected by cavitary plaques $(P=.003), 90.3 \%$ involving the anteroinferior wall, and $3.2 \%$, the posterior wall. Involvement of the anteroinferior IAC as the most common location is consistent with reports in the literature. ${ }^{8,10}$ In this location, cavitary plaques have been called "cavitary formations," "cavitations," and diverticula or indentations of the IAC. ${ }^{1,7-10}$ We found 1 temporal bone with a cavitary plaque involving the posterior wall of the IAC in a patient with advanced otospongiosis (grade 3) and sensorineural hearing loss. However, the preference for this site in this patient is uncertain. Cavitary plaques outside the IAC were seen in 2 instances (Fig 3). These locations are rare and have been previously documented in 4 case reports, most of them identified on histologic analysis. ${ }^{1,9,16,17}$

Cavitary plaques showed low attenuation on CT, similar to that of CSF in the IAC (Fig 1). We found that there was a statistically significant difference in the mean Hounsfield units between the noncavitary plaques and cavitary lesions, $953 \pm 278$ versus $115 \pm 75$, respectively $(P<.001)$. This difference in Hounsfield units was expected because $96 \%$ of cavitary plaques were in apparent communication with the IAC and presumably filled with CSF (Fig 2). This finding was also demonstrated in a patient with cavitary otospongiosis who underwent MR imaging, which serves as an illustrative example (Fig 2C). Cavitary plaques may be difficult to evaluate on imaging due to their small size. Therefore, in patients with suspected otospongiosis, it is important to scruti- 
nize the anteroinferior wall of the IAC next to the cochlea, which is where cavitary changes are most commonly identified.

Some unrelated disorders affecting the labyrinth can produce third window lesions, resulting in conductive or sensorineural hearing loss, vestibular manifestations (sound and/or pressureinduced vertigo), or a combination. ${ }^{18,19}$ Cavitary plaques have been described as a cause of third window lesions when they reach the endosteal margin of the bony labyrinth. ${ }^{1,16,20}$ However, this complication is probably rare because $90 \%$ of the cavitary plaques in our study did not show extension into the endosteal layer of the cochlea. Three cavitary plaques showed contact with the endosteal margin of the basal turn of the cochlea, but none of them had clinical manifestations of third window phenomena. It is possible that involvement of the endosteal layer in these cases was too mild to result in third window abnormalities.

Pippin et $\mathrm{al}^{8}$ demonstrated a significant correlation between the presence of cavitary plaques and isolated sensorineural hearing loss. In our study, the presence of cavitary plaques was not statistically associated with a specific type of hearing loss $(P=$ .365); however, this finding could be related to our smaller sample size. Also, there was probably an effect of patient selection because their cohort included many patients with cavitary changes but without classic findings of otospongiosis.

Complications of cochlear implantation in patients with otospongiosis are reported to occur in $10 \%-20 \%$ of patients. ${ }^{21,22}$ Cavitary plaques as a potential cause of CSF gushing and misplacement of electrode arrays into the pericochlear cavities have been reported in around 4 cases in the literature. Otospongiosis leads to loss of part of the wall of the cochlea, which can result in direct communication between the IAC and the basal turn. ${ }^{1,15,21,22}$

In our patients, we found 6 temporal bones with cavitary plaques that underwent cochlear implantation. These cases showed a discrete layer of intervening bone between the cavitary plaques and the basal turn of the cochlea, and as expected, none of them had any complications related to the operation. The absence of complications in our study, however, could also be explained by the limited number of temporal bones that underwent a cochlear implantation.

The limitations of our study include its retrospective nature, relatively small sample size, and absence of pathologic confirmation because biopsies are not routinely performed during stapedectomy. However, all patients met imaging and/or clinical criteria for otospongiosis. Measurement of Hounsfield units could have also been affected by the small size of the lesions and partial averaging with adjacent bone.

\section{CONCLUSIONS}

Cavitary plaques in otospongiosis were seen in one-third of temporal bones, and their most common location was the anteroinferior wall of the IAC next to the cochlea. Cavitary plaques were seen mostly in older patients, and there was no association between them and a greater degree of otospongiosis by imaging or third window manifestations. There were no procedural complications such as CSF gusher or misplacement of electrodes within cavitary plaques during cochlear implantation.

Disclosures: Feng-Chang Lin—RELATED: Grant: National Institutes of Health, Comments: ULITR001111*; UNRELATED: Employment: University of North Carolina at Chapel Hill. *Money paid to the institution.

\section{REFERENCES}

1. Makarem AO, Hoang TA, Lo WW, et al. Cavitating otosclerosis: clinical, radiologic, and histopathologic correlations. Otol Neurotol 2010;31:381-84 CrossRef Medline

2. Clayton AE, Mikulec AA, Mikulec KH, et al. Association between osteoporosis and otosclerosis in women. J Laryngol Otol 2004;118: 617-21 Medline

3. Redfors YD, Möller C. Otosclerosis: thirty-year follow-up after surgery. Ann Otol Rhinol Laryngol 2011;120:608-14 CrossRef Medline

4. Palacios E, Valvassori G. Cochlear otosclerosis. Ear Nose Throat J 2000;79:494 Medline

5. Wycherly BJ, Berkowitz F, Noone AM, et al. Computed tomography and otosclerosis: a practical method to correlate the sites affected to hearing loss. Ann Otol Rhinol Laryngol 2010;119:789-94 CrossRef Medline

6. Sanverdi SE, Ozgen B, Dolgun A, et al. Incomplete endochondral ossification of the otic capsule, a variation in children: evaluation of its prevalence and extent in children with and without sensorineural hearing loss. AJNR Am J Neuroradiol 2015;36:171-75 CrossRef Medline

7. Bou-Assaly W, Mukherji S, Srinivasan A. Bilateral cavitary otosclerosis: a rare presentation of otosclerosis and cause of hearing loss. Clin Imaging 2013;37:1116-18 CrossRef Medline

8. Pippin XK, Muelleman XT, Hill XJ, et al. Prevalence of internal auditory canal diverticulum and its association with hearing loss and otosclerosis. AJNR Am J Neuroradiol 2017;38:2167-71 CrossRef Medline

9. Makarem AO, Linthicum FH. Cavitating otosclerosis. Otol Neurotol 2008;29:730-31 CrossRef Medline

10. Hoeberigs M, Postma A, Waterval J, et al. Prevalence of anterior internal auditory canal "diverticulum" on high resolution CT in patients with otosclerosis. In: Proceedings of the Scientific Assembly and Annual Meeting of the Radiological Society of North America, Chicago, Illinois. November 25-30, 2012

11. Lee TC, Aviv RI, Chen JM, et al. CT grading of otosclerosis. AJNR Am J Neuroradiol 2009;30:1435-39 CrossRef Medline

12. Merkus P, van Loon MC, Smit CF, et al. Decision making in advanced otosclerosis: an evidence-based strategy. Laryngoscope 2011; 121:1935-41 CrossRef Medline

13. Schuknecht HF, Kirchner JC. Cochlear otosclerosis: fact or fantasy. Laryngoscope 1974;84:766-82 Medline

14. Brondbo K, Hawke M, Abel SM, et al. The natural history of otosclerosis: a correlation of the volume and activity of the otosclerotic lesion with age. J Otolaryngol 1983;12:163-68 Medline

15. Ramsden R, Bance M, Giles E, et al. Cochlear implantation in otosclerosis: a unique positioning and programming problem. $J$ Laryngol Otol 1997;111:262-65 Medline

16. Richard CC, Linthicum FH Jr. An unexpected third window in a case of advanced cavitating otosclerosis. Otol Neurotol 2012;33:e47-48 CrossRef Medline

17. Hederstierna C, Cureoglu S, Paparella MM. Undiagnosed severe cochlear otosclerosis as a cause of profound hearing loss. Otol Neurotol 2013;34:14-15 CrossRef

18. Merchant SN, Rosowski JJ. Conductive hearing loss caused by thirdwindow lesions of the inner ear. Otol Neurotol 2008;29:282-89 CrossRef Medline

19. Ho ML, Moonis G, Halpin CF, et al. Spectrum of third window abnormalities: semicircular canal dehiscence and beyond. AJNR Am J Neuroradiol 2017;38:2-9 CrossRef Medline

20. Quesnel AM, Moonis G, Appel J, et al. Correlation of computed tomography with histopathology in otosclerosis. Otol Neurotol 2013;34:22-28 CrossRef Medline

21. Rotteveel LJ, Proops DW, Ramsden RT, et al. Cochlear implantation in 53 patients with otosclerosis: demographics, computed tomographic scanning, surgery, and complications. Otol Neurotol 2004; 25:943-52 CrossRef Medline

22. Ramsden R, Rotteveel L, Proops D, et al. Cochlear implantation in otosclerotic deafness. Adv Otorhinolaryngol 2007;65:328-34 Medline 